\title{
Author Correction: Non-flammable electrolyte enables Li-metal batteries with aggressive cathode chemistries
}

Xiulin Fan, Long Chen, Oleg Borodin, Xiao Ji, Ji Chen, Singyuk Hou, Tao Deng, Jing Zheng, Chongyin Yang, Sz-Chian Liou, Khalil Amine, Kang Xu and Chunsheng Wang (D)

Correction to: Nature Nanotechnology https://doi.org/10.1038/s41565-018-0183-2, published online 16 July 2018.

In the version of this Article originally published, in the first paragraph of the Methods, HFE was incorrectly given as 2,2,2-Trifluoroethyl$3^{\prime}, 3^{\prime}, 3^{\prime}, 2^{\prime}, 2^{\prime}$-pentafluoropropyl ether; it should have been 1,1,2,2-tetrafluoroethyl- $2^{\prime}, 2^{\prime}, 2^{\prime}$-trifluoroethyl ether. This has now been corrected in the online versions of the Article.

Published online: 4 October 2018

https://doi.org/10.1038/s41565-018-0293-x

\section{Author Correction: Subwavelength angle-sensing photodetectors inspired by directional hearing in small animals}

Soongyu Yi, Ming Zhou D , Zongfu Yu, Pengyu Fan, Nader Behdad, Dianmin Lin (D), Ken Xingze Wang, Shanhui Fan and Mark Brongersma

Correction to: Nature Nanotechnology https://doi.org/10.1038/s41565-018-0278-9 published online 29 October 2018.

In the version of this Letter originally published, Zongfu Yu was mistakenly not noted as being a corresponding author; this has now been corrected in all versions of the Letter.

\section{Publisher Correction: Anisotropic polarization-induced conductance at a ferroelectric-insulator interface}

Yi Zhang DiD, Haidong Lu, Lin Xie, Xingxu Yan, Tula R. Paudel, Jeongwoo Kim, Xiaoxing Cheng, Hui Wang (D,

Colin Heikes D, Linze Li, Mingjie Xu, Darrell G. Schlom, Long-Qing Chen, Ruqian Wu, Evgeny Y. Tsymbal,

Alexei Gruverman (D) and Xiaoqing Pan

Correction to: Nature Nanotechnology https://doi.org/10.1038/s41565-018-0259-z, published online 24 September 2018.

In the version of this Letter originally published, the right-hand arrow in Fig. $3 \mathrm{~b}$ was incorrectly labelled $[\overline{1} 0]_{0}$; it should have been $[\overline{1} 01]_{0}$; this has now been corrected. Also, ref. 29 was incorrectly included in the reference list; it has now been removed. 